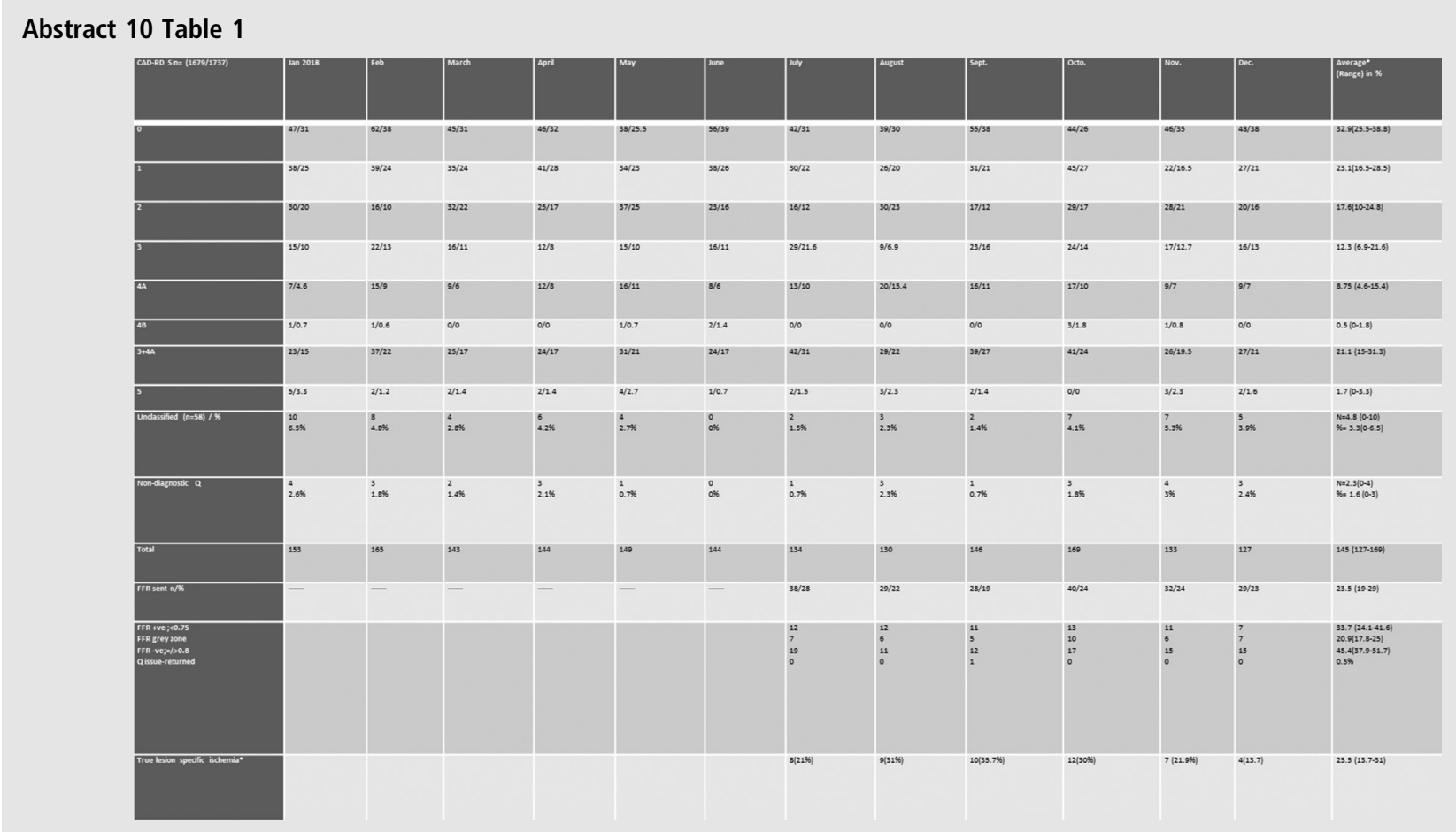

regarding the potential need of further assessment and management. Yet an important and welcome 'by-product' may be the ability to respectively quantitate and semi-quantitate the extent and/or severity of disease biomarkers in large distinct patient groups and population. For example, characterisation of the UK NICE-CG95 compliant RACPAC populations, as underway in the National audit CA2017-18-191, has the potential to yield extremely valuable information on disease distribution and hot-spots allowing the creation of 'disease heat maps' which in turn can be used to inform public health and research strategies alike as well as informing and ultimately optimising down-stream resource utilisation.

Methods We sought to characterise our own RACPAC population and determine to what degree the variability in disease over time may be influenced by intra- and inter-observer variability. This is a basic yet important first step to understand fluctuations of disease prevalence and variation. We studied our RACPAC-CT data with respect to CAC-RDS and CADRDS reporting output over a 1-year period. We performed inter- and intra-observer variability for both CAC-RDS and CAD-RDS of the 2 Consultants with the highest reporting volume (>500 reports/annum). Below we show the completed data relating to the 2018 Calendar year submitted to the national audit.

Results Below Table 1 shows the 2018 CAD-RDS disease distribution of the Manchester Foundation Trust (Wythenshawe site) Cardiac CT service which is fed by the RACPAC clinics of Wythenshawe, Stepping Hill Hospital FT and Salford Royal FT. Cohen's Kappa analysis for CAC-RDS and CAD-RDS intra and inter-observer variability for 20 randomly selected cases within were as follows;

CAC-RDS- intra-observer variability; Weighted Kappa 1.0 (95\% CI 1.0-1.0)

$\mathrm{CAC}=\mathrm{RDS}$ - inter-observer variability; Weighted Kappa 0.96 (95\% CI $0.90-1.0$ )

CAD-RDS - intra-observer variability; Weighted Kappa 0.84 (95\% CI $0.715-0.98)$
CAD-RDS - inter-observer variability; Weighted Kappa 0.9 (95\% CI 0.80-0.99)

Conclusion This QC process of real-world data collected in a NICE CG95 compliant NHS environment demonstrates that the disease population presenting to GM RACPAC clinics is reasonable stable over time and broadly similar to the population studied in SCOTHEART. It provides sound data for commissioners and healthcare providers with respect to the disease distribution and severity presenting via NICE CG 95 compliant RACPAC clinics. Importantly, it demonstrates that temporal disease variability is unlikely the consequence of significant variability of CAC-RDS and CAD-RDS application and variation when applied by experienced practitioners and as such validates these tools to compare regional and national data.

\section{EVALUATION OF TUBE POTENTIAL EFFECTS ON ATHEROSCLEROTIC PLAQUE ASSESSMENT: IN VIVO ASSESSMENT WITH INTRAVASCULAR ULTRASOUND} ${ }^{1,2}$ Anantharaman Ramasamy, ${ }^{1,2}$ Francesca Pugliese,
Horvat, ${ }^{1,2}$ Anthony Mathur, ${ }^{1,2}$ Andreas Baumbach, ${ }^{1,2,4}$ Christos V Boon, ${ }^{4}$ Pal Mantas. ${ }^{1}$ Departmentch-
of Cardiology, Barts Heart Centre, Barts Health NHS Trust, London, UK; ${ }^{2}$ William Harvey
Research Institute, Queen Mary University London, UK; ${ }^{3}$ Institute of Cardiovascular Sciences,
University College London, London, UK; ${ }^{4}$ MTA-SE Lendulet Cardiovascular Imaging Research
Group, Heart and Vascular Centre, Semmelweis University, Budapest, Hungary

\subsection{6/heartjnl-2019-BSCI.11}

Introduction Computed tomographic coronary angiography (CTCA) is a non-invasive imaging modality which allows plaque burden $(\mathrm{PB})$ and composition assessment. Lowering the tube potential (kilovolt $[\mathrm{kV}]$ ) is key strategy to reduce radiation dose to patients. In vivo studies have not investigated the effects of lower tube potential $(<120 \mathrm{kV})$ for plaque assessment. We aim to evaluate the effects of different $\mathrm{kVs}$ on plaque burden assessment in comparison to intravascular imaging. 
Methods 11 patients with stable angina were prospectively recruited for a third generation dual-source CTCA (prospective ECG-triggered sequential scans, gantry rotation $250 \mathrm{~ms}$, $128 \times 2 \times 0.5 \mathrm{~mm}$ collimation with tube voltage $(\mathrm{kV})$ and current determined by scanner) and 3-vessel intravascular imaging (IVUS). The IVUS pullbacks were manually segmented and plaques with $\mathrm{PB}>40 \%$ were included in the study. Semiautomated CTCA segmentation of lumen and vessel wall borders were performed and corresponding plaques with IVUS were identified. The PB at the minimum lumen area in CTCA and IVUS were estimated and compared.

Results The mean age of the studied population was $61 \pm 11$, $3(27.2 \%)$ were diabetic and $7(63.6 \%)$ suffered from hypercholesterolaemia. Group $1(<100 \mathrm{kV})$ included 6 patients $(16$ plaques) while group $2(\geq 100 \mathrm{kV})$ included 5 patients (11 plaques). CTCA-derived PB was similar between both groups

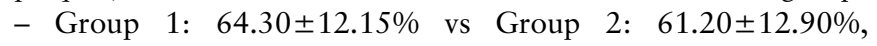
$\mathrm{p}=0.551$. However, Group 2 showed better correlation between IVUS and CTCA-derived PB $(68.00 \pm 13.58 \%$ vs $61.20 \pm 12.90 \%, \mathrm{r}=0.863, \mathrm{p}=0.006)$ compared to Group 1 $(59.79 \pm 9.66 \% \quad$ vs $\quad 64.30 \pm 12.90 \%, \quad \mathrm{r}=0.222, \quad \mathrm{p}=0.427)$, respectively.

Conclusion Higher tube voltage threshold $(\geq 100 \mathrm{kV})$ allows more accurate PB assessment in CTCA.

\section{A STANDARDISED NUMERICAL FFRCT REPORTING PROPOSAL TO PRAGMATICALLY GUIDE APPROPRIATE CATHETER LABORATORY USE WITHIN A NICE COMPLIANT CHEST PAINS PATHWAY}

Matthias Schmitt. Manchester University NHS Foundation Trust, UK

\subsection{6/heartjnl-2019-BSCI.12}

Background As per NICE CG 95 11/2016 iteration Cardiac $\mathrm{CT}$ is now the first line investigation for patients with stable chest pains of assumed cardiac origin. NICE (MTG 32) also endorses the use of Heartflow FFRCT assessment for studies with intermediate lesion severity. The introduction of CACRDS and CAD-RDS reporting is facilitating a standardised reporting and provides both recommendations for appropriate initiation of preventive therapy and appropriate further assessment respectively. However, there is no general consensus on how to integrate the output of Heartflow FFRCT assessment into a coherent reporting structure.

Objective To provide, similar to CAC-RDS and CAD-RDS, a pragmatic standardised FFRCT reporting scheme which emphasises the presence of Lesion specific ischaemia (LSI) in the context of the lesion location over and above the sole numerical FFR value adjudication in order to enhance the role of Cardiac CT as a tool to appropriately enrich the catheter laboratory population and aide clinical decision making following CCTA+FFRCT.

Methods The disease spectrum of 196 consecutive FFRCT studies acquired over a 6 month period in a single UK centre was reviewed and subsequently graded according to; 1) the lowest FFRCT in any analysable vessel as negative (FFRCT $>0.8$ ), grey zone $(0.8 \geq$ FFRCT $>0.75)$, positive (FFRCT $<0.75)$. 2) The number (Nr) of affected coronaries (1-4). 3) The presence/absence of LSI defined as localised (within a proximal or mid segment) pressure drop and subdivided in + ve $(++)$ and grey zone LSI $(+)$. 4) The location of the LSI, i.e. proximal (A) coronary segments (SCCT segments 1,5,6,11), mid (B) segments and proximal side-branches (SCCT segments $2,3,7,9,12,13,17$ ), and distal (C) segments (SCCT segments $4,8,14,15,16,18)$. Using these criteria a simple numerical scoring system was developed which may aide pragmatic clinical decision making. Exemplar illustration is provided below.

Results Out of 839 patients 196 (23.3\%) were CAD-RDS 3/ 4A and were sent for FFRCT analysis. Of those 196 patients $33.7 \%$ were FFRCT +ve (FFRCT <0.75), 20.9\% were grey zone FFR (0.75-0.799) and 45.4\% were FFRCT negative (FFRCT $=>0.8)$. One scan $(0.5 \%)$ was returned for quality reasons (image motion). LSI as defined above occurred in 50 scans $(25.5 \%)$.

Conclusion Here we present a proposal of standardised FFRCT scoring, which categorises the presence of LSI and
- -ve FFRCT $(=/>0.8)$ in all vessels

- Grey zone FFRCT (0.75-0.79)

- Nr affected vessels $1 / 2 / 3 / 4$;

- LSI present/absent; $+/-$;

- Lesion location; $\left(\mathrm{A}^{*} / \mathrm{B}^{*} / \mathrm{C}^{*}\right)$;

- NoLSI

- +ve $(<0.75)$

- $\mathrm{Nr}$ affected vessels $1 / 2 / 3 / 4$

- LSI present/absent; ++/- [FFRCT<0.75]

- LSI grey zone; +/- [FFR0.75-0.79]

- Lesion location; $\left(A^{*} / B^{*} / C^{*}\right)$

- No LSI

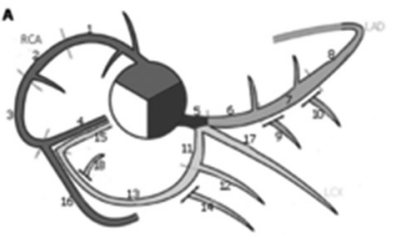

Additive Score

scores 1

2 vessels score $1,>2$ scores 2

+ in A scores 2, + in B scores 1

scores 2

2 vessels score $1,>2$ scores 2

++ in $A$ and $B$ score 2

+ in $A$ and $B$ scores 1

Score 0-2; Favours conservative

management, IA unlikely to lead to revascularisation.

Score 3; Consider stress echo/FI

Score $>\mathbf{3}$; Invasive coronary angiography

likely appropriate and increasingly high

likelihood of revascularisation

${ }^{*} \mathrm{~A}$; Proximal (segments $1,5,6,11$ ), ${ }^{*} \mathrm{~B} / \mathrm{mid} 2,3,7,9,13,17,12,{ }^{*} \mathrm{C}$ distal coronary segment $4,16,14,15,18,8$

Abstract 12 Figure 1 Examples 